\title{
A Design Reuse Method of 3D CAD Model Based on Retrieval
}

\author{
Zhang Kaixing ${ }^{1, a}$, Hang Shengyu ${ }^{1, b}$, Liu Xianxi ${ }^{1,2, c, *}$ \\ ${ }^{1}$ College of Mechanical and Electronic Engineering, Shandong Agricultural University, Tai'an, \\ China; ${ }^{2}$ Shandong Provincial Key Laboratory of Horticultural Machineries and Equipments, Tai'an, \\ China \\ a email: kaixingzhang@139.com, ${ }^{\mathrm{b}}$ email: sdaujdxyhsy@163.com, ${ }^{* c}$ email: bullish@sdau.edu.cn
}

Keywords: Design reuse, 3D CAD model, Model retrieval

Abstract. With respect to the requirement of precise and intelligent design reuse in product digital design, this paper proposes a novel 3D model retrieval method for product design reuse, the method use the design feature as the carriers to organize the geometric and design feature information. First, the concept of the design feature model is proposed, the 3D CAD model is featured and represented using a feature attribute adjacency graph, and a CAD models is represented as a set of the design features. After that the sub-parts contained in the 3D models are regarded as the nodes with attributes. Accordingly, each CAD model can be represented as a set of the nodes, and the comparison of two models is transformed into similarity measuring their two corresponding sets of nodes. At last, we complete the similarity assessment by using an optimal matching algorithm for bi-graphs on the comparison of the similarity of the model nodes, and realize related model and design resource effective reuse. Experimental results show the proposed method can achieve the 3D model retrieval and support the design reuse.

\section{Introduction}

With the development of digital design and manufacturing integrated technology, 3D CAD model has become the core media of expressing product design and manufacturing information. As the number of 3D models accumulated by enterprises in the process of production is continuously increasing, how to efficiently locate the desired model, fully exploit and reuse the implicit knowledge, have an important role to improve the mechanical design efficiency and quality in the product design field.

Generally speaking, what we need to do first in the semantic retrival of 3D models is to split these models to make them represented with different sub-structures. Then we can achieve the semantic retrieval process through the descriptions and annotations to models ${ }^{[1][2]}$. Razdan ${ }^{[3]}$, etc. adopted an algorithm based on the watershed to achieve the segmentation of 3D models, carrying out corresponding editing and semantic annotation to the segmentations. Gal ${ }^{[4]}$, etc. used the curvature's difference of the model's surface to achieve the automatic segmentation of different models and evaluate the overall similarity of model through local structure's similarity. Funkhouser ${ }^{[5]}$, etc. evaluated the overall similarity of model by using the method of constructing the 
local region. Research institutions in Europe, the United States and other countries ${ }^{[6][7]}$ decomposed the 3D models into meaningful subparts based on the perceptual characteristics of the models, and semantic tags to each sub-section, constituting the semantic representation of the model to achieve the model retrieval and reuse.

This paper proposes a novel 3D model retrieval method for product design reuse, the method uses the design feature as the carriers to organize the geometric and design feature information.

\section{The definition and representation of the structural CAD model}

The structured CAD model is mainly constituted by the geometric information, topology information, and feature information. Geometric information refers to the geometry of the CAD model. Topological information refers to the topology adjacency of CAD models. Feature information refers to the features or typical structures (combination of features) CAD model contained. The internal relations of the structural CAD model are described by the features and adjacent diagrams of typical structures, the graph vertices represent characteristics or typical structures, vertex has the attribute information, vertex of connections between adjacent relations representative between them, and the connections between the vertices represent the adjacency relationship between them. Adjacency graph can not only present the CAD model in a structured form, but also give the distribution of features/characteristic structures in CAD model and the location of mutual positional relationship.

Structural CAD model is a collection of characteristics or typical structures, feature mainly refers to the basic manufacturing feature, and the typical structures are multiple feature combination areas with reuse value, and characteristic and the typical structures have parameterized geometric information.

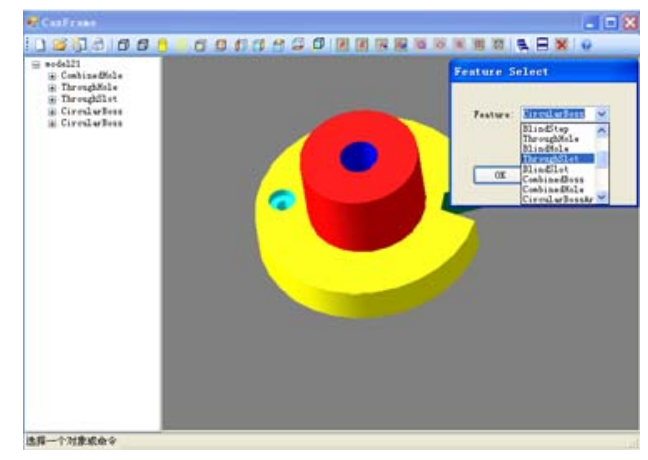

Figure 1 Model Feature Recognition

In order to realize the representation of the structured CAD model, 3D CAD models need to be represented as a collection of features / characteristic structures. First of all, using an algorithm combined diagrams and rules to realize automatic feature recognition ${ }^{0[9]}$. To the recognition of intersecting features, the algorithm has good effect and high recognition efficiency. Few amount characteristics which are difficult to automatically identify use human-computer interaction to achieve, as shown in Figure 1.The characteristics considered in this paper are basic manufacturing features and composite characterized by combinations of these basic characteristics, made or arranged according to certain rules and arrays, basic features include the following five categories: boss, pocket, step, hole and slot. Then, based on the characteristic representation of CAD model, discovering and extracting the typical structure CAD model contained, extracting 24 typical structures from the model library. Through the above steps, the CAD model is represented as a collection of features / typical structures. 
Similarity assessment of structural CAD model includes the type, shape information, topological connection information and scale information. Similarity assessment of model combines the geometric information, topological information and feature information of model, fitting the requirement of semantic retrieval reuse better.

(1) Comparison of different types

In the comparison of characteristics / typical structures' geometric feature, if the types of two features / typical structures being compared are different, that their similarity is 0 , and if they are the same types, that their similarity is 1 . Set the type of two compared features / typical structures as $T_{1}$ and $T_{2}$, and similarity of types use the $S_{T}$ to express, as shown in equation (1).

$$
S_{T}= \begin{cases}0 & T_{1} \neq T_{2} \\ 1 & T_{1}=T_{2}\end{cases}
$$

(2) Comparison of the shape information

For features/characteristic structures whose types are the same, shape differences may exist. In order to obtain the differences of geometry information, this article will use the shape distribution algorithm to compare geometric information of characteristic/typical structures. This method has the advantages of simple calculation and good robustness. Randomly selecting points on the surface of the model, then count the distance of random point (D2) as a measurement, formatting the distribution curve, and through the EMD distance ${ }^{0}$ to compare the shape distribution curves, so as to realize the comparison of characteristics / typical structures' geometry information. Set the distance between the two characteristic/typical structures' distribution curves compared to be EMD (X, Y), then the similarity $S_{D}$ of their shape information will be:

$$
S_{D}=1-E M D(X, Y)
$$

(3) Comparison of topology connection information

Topological structure plays an important role in the similarity evaluation of the model, topological similarity between two features/characteristic structures use the figure (3) to calculate. Wherein: $n_{e}$ represents the number of compatible edges between the two characteristics / typical structures, and $n_{\mathrm{T}_{1}}, n_{\mathrm{T}_{2}}$ represent the number of the partial structures adjacent to the two compared characteristics / typical structures.

$$
S_{L}=\frac{n_{e}}{\max \left(n_{\mathrm{T}_{1}}, n_{\mathrm{T} 2}\right)}
$$

(4) Comparison of the scale information

For features/characteristic structures of the same shape type, different scales may lead to its reuse value completely different. For example, the apertures can be processed using the method of drilling, while large holes need Boring, their processing methods are different. Scale similarity between the two features / typical structures is evaluated using their minimum bounding box. Set the minimum bounding box of the two compared characteristics / typical structures length, width and height, respectively $a 1, a 2, a 3$ and $b 1, b 2, b 3$, then their scale similarity can be calculated in the formula (4). 


$$
S_{Q}=\sum_{i=1}^{3}\left(1-\frac{\left|a_{i}-b_{i}\right|}{\max \left(a_{i}, b_{i}\right)}\right) / 3
$$

Similarity of the features/characteristic structures of T1 and T2 can be obtained by its type, shape similarity, topological similarity and scale similarity in the comprehensive weighted method, and expressed with $\delta$, then:

$$
\delta=\left\{\begin{array}{cc}
0 & T_{1} \neq T_{2} \\
\omega_{T} S_{T}+\omega_{\mathrm{D}} S_{\mathrm{D}}+\omega_{\mathrm{L}} S_{\mathrm{L}}+\omega_{\mathrm{Q}} S_{Q} & T_{1}=T_{2}
\end{array}\right.
$$

$\omega_{T}, \omega_{\mathrm{D}}, \omega_{\mathrm{L}}$ and $\omega_{\mathrm{Q}}$ respectively represent the weight coefficient of each component, and their sum is 1 .

Regard each features/characteristic structures of the structural CAD model as a node, and regarded the type, shape information, topology information, and scale information of characteristics / typical structures as the attributes of the node. The similarity assessment is completed by using an optimal matching algorithm for bi-graphs on the comparison of the similarity of structural model nodes.

Set the collection of nodes the structural CAD model M1 contained can be expressed as $T_{1}=$ $\left\{\mathrm{t}_{11}, \mathrm{t}_{12} \ldots \mathrm{t}_{1 \mathrm{~m}}\right\}$, nodes collection of $\mathrm{M} 2$ can be expressed as $T_{2}=\left\{\mathrm{t}_{21}, \mathrm{t}_{22}, \ldots, \mathrm{t}_{2 \mathrm{n}}\right\}$, similarity comparison of model is transformed into optimal matching problem of complete bipartite graph consisting of two group of nodes. Construction of complete bipartite graph is shown in Figure 3, set the complete bipartite graph $G=(V, E)$, in the formula, the vertices of a graph $V$ are the union of node setT1 and $\mathrm{T} 2, V=T_{1} \cup T_{2}$. And collection connection relationship between set $\mathrm{T} 1$ and set $\mathrm{T} 2$ in each node doesn't exist, for any node pairs $t_{1 \mathrm{i}} \in T_{1}$ and $t_{2 \mathrm{j}} \in T_{2}$, connecting them with an edge.

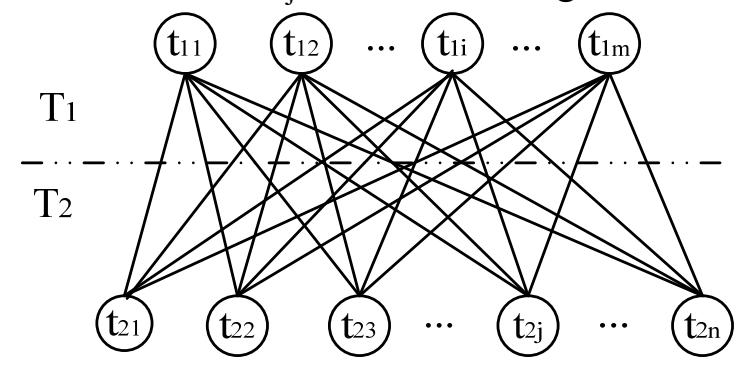

Figure 2 Construct Figure of Bi-graphs

To any node pairs of two models, according to the node attributes, similarity coefficient for each node can be calculated using equation (5) and set the similarity coefficient as two bipartite graphs' weights, Then the weights matrix of the bipartite graph is the similarity matrix between the two structural CAD model, as shown in equation (6), when the number of two node groups is not the same, pad 0 , make the similarity matrix square. 


$$
\begin{gathered}
t_{11} \\
t_{12} \\
\ldots \\
t_{1 i} \\
\ldots \\
t_{1 m} \\
0 \\
0
\end{gathered}\left[\begin{array}{cccccccc}
\delta_{11} & t_{22} & t_{23} & \ldots & t_{2 j} & \ldots & t_{2 n} \\
\delta_{21} & \delta_{22} & \delta_{23} & \ldots & \delta_{2 j} & \ldots & \delta_{2 n} \\
\ldots & \ldots & \ldots & \ldots & \ldots & \ldots & \ldots \\
\delta_{i 1} & \delta_{i 2} & \delta_{i 3} & \ldots & \delta_{i j} & \ldots & \delta_{i n} \\
\ldots & \ldots & \ldots & \ldots & \ldots & \ldots & \ldots \\
\delta_{m 1} & \delta_{m 2} & \delta_{m 3} & \ldots & \delta_{m j} & \ldots & \delta_{m n} \\
0 & 0 & 0 & 0 & 0 & 0 & 0
\end{array}\right]
$$

In order to get overall optimal matching of the two compared structural CAD model between each local structure, Kuhn-Munkres algorithm which is the best in calculating optimal matching of the two graphs is used to calculate the optimum matching scheme of similarity matrix. According to the calculated optimal matching scheme, similarity of two models will be evaluated with the values of optimal matching areas.

The more features each node of structural CAD model contained, the more effects on model similarity. Therefore, considering the influence of the number of features the different partial structure contained to the model similarity, and the similarity values of the two models will be calculated using equation (7).

$$
S=\sum_{j=1}^{n}\left(\frac{f_{m(j)}+f_{j}}{2} \cdot \delta_{m(j) j}\right) / \max \left(\sum f_{m(j)}, \sum f_{j}\right)
$$

Wherein, $m_{j}$ represents the number of rows that can make an optimal matching with the $j$-th column in formula $(5), \delta_{m(j) j}$ is the similarity coefficient of the $m(j)$-th partial structure of the model $M_{1}$ and the m-th partial structure of model M2, $f_{m(j)}$ represents the number of features the $m(j)$-th partial structure of the model $M_{1}$ contained, $f_{j}$ represents that the $j$-th partial structure of the model M2 contained.

\section{Experimental results and analysis}

In order to verify the effectiveness of the algorithm, Microsoft Visual Studio 2008 is used as the integrated development environment, and Open CASCADE is adopted as the geometry modeling platform. Model library contains about 200 common 3D CAD models and 52 features/characteristic structures.

Table 3 show the experimental results of the algorithm in this chapter, shape distribution algorithm ${ }^{[10]}$ and spherical harmonics algorithms ${ }^{[12]}$ applying to two CAD models retrieval respectively. Shape distribution algorithm and spherical harmonics algorithm evaluate the models from the similarity of geometric similarity, and the algorithm in this chapter takes the geometric model, topological information, structure information and scale information into account. Therefore, it is able to reuse the design information better, and better in line with the needs of CAD domain semantic retrieval. 
Table 1 Retrieve Example 1

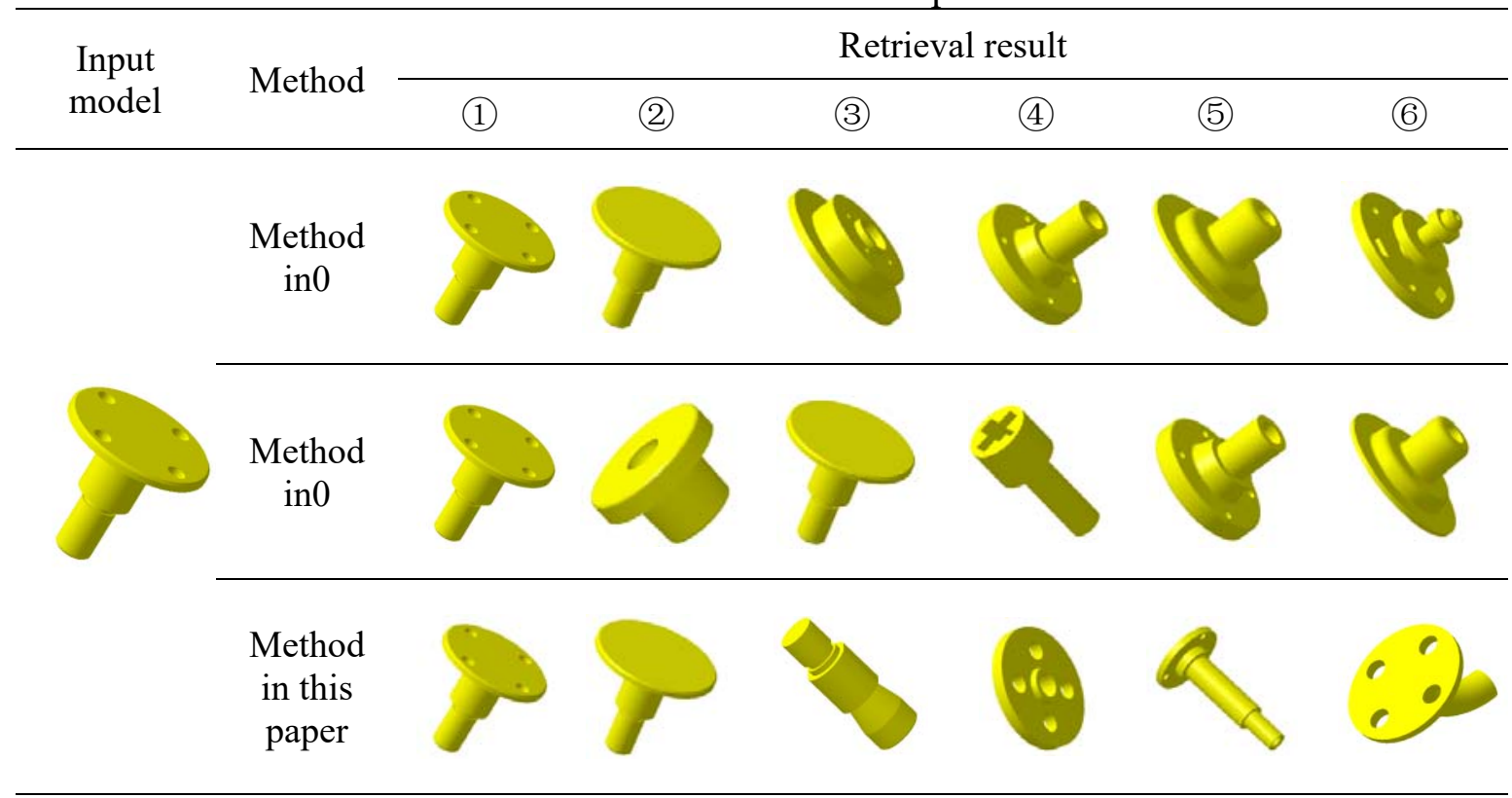

\section{Conclusion}

We propose the concept of the structural CAD model, intially represent a CAD models as a set of the features and the characteristic structures which indicate the manufacturing semantic information, and then represent the structural CAD model by using the ontology technique. After that we take the attribute information of the structural CAD models into consideration, and extract them in retrieval. The sub-parts contained in the structural CAD models are regarded as the nodes with attributes. Accordingly, each CAD model can be represented as a set of the nodes, and the comparison of two models is transformed into similarity measuring their two corresponding sets of nodes. At last, we complete the similarity assessment by appling an optimal matching algorithm for bi-graphs on the comparison of the similarity of structural model nodes. Due to the structure-based representation in similarity assessment, the proposed method can better satisfied the demands in CAD semantic retrieval, and is of great importance significance in the semantic retrieval and reuse.

\section{References}

[1] Paquet, Rioux. A query by content software for three dimensional databases management. In: Proceedings of the International Conference on Recent Advances in 3D Digital Imaging and Modeling, 1997. 345-352.

[2] Pan Xiang, Zhang Sanyuan, Ye Xiuzi. A Survey of Content-based 3D Model Retrieval with Semantic Features[J]. Chinese Journal of Computers, 2009, 32(6): 1069 1079.

[3] Razdan A, Rowe J, Tocheri M, et al. Adding semantics to 3D digital libraries[C]. Proceedings of the International Conference on Asian Digital Libraries, Singapore, 2002: 419 420.

[4] Gal R, Cohen-Or D. Salient geometric features for partial shape matching and similarity [OL].

[5] Funkhouser T, Shilane P. Partial matching of 3D shapes with priority-driven search [OL].

[6] Attene M, Robbiano F, Spagnuolo M. Part-based annotation of virtual 3D shapes[C]. Proceedings of the International Conference on Cyberworlds. Hannover, Germany, 2007: 427 436.

[7] Gutierez M, Garca-Rojas A, Thalmann D. An ontology of virtual humans incorporating semantics into human shapes [J]. The Visual Computer, 2007, 23 (3): 207 218. 
[8] Sunil VB, Agarwal R, Pande SS. An approach to recognize interactiong features from B-Rep CAD models of prismatic machined parts using a hybrid (graph and rule based) technique[J]. Computer in Industry, 2010, 61(7):686 701.

[9] Zhang Kaixing, Zhang Shusheng, Bai Xiaoliang. Automatic Extraction of Common Reusable Partial Structures in 3D CAD Models [J]. Journal of Computer-Aided Design \& Computer Graphics, 2011, 23(9): $1512 \sim 1519$.

[10] Osada R, Funkhouser T, Chazelle B, et al. Shape distributions [J].ACM Transactions on Graphics, 2002, 21(4), 807 832.

[11] Zhao Q, Tao H. Differential earth mover's distance with its application to visual tracking[J]. IEEE Transactions on Pattern Analysis Machine Intelligence, 2010, 32(2): 274 287.

[12] Kazhdan M, Funkouser T, Rusinkiewicz S. Rotation invariant spherical harmonic representation of 3D shape descriptors [C]. Proceedings of Symposium on Geometry Processing, Aachen, 2003: 156 164. 